\title{
Comida de rua e intervenção: estratégias e propostas para o mundo em desenvolvimento
}

\author{
Street food and intervention: \\ strategies and proposals to the developing world
}

Ryzia deCassia Vieira Cardoso ${ }^{1}$

Sandra M aria Chaves dos Santos ${ }^{2}$

Edleuza OliveiraSilva ${ }^{3}$

${ }^{1}$ Departamento deCiências dosAlimentos, Escola de Nutrição, UFBA. Rua Araújo Pinho 32, Canela. 40110-150 Salvador BA. ryzia@ufba.br

${ }^{2}$ Departamento deCiências da Nutrição, Escola de Nutrição, UFBA.

${ }^{3}$ Vigilância Sanitária, Secretaria M unicipal de SaúdedeSalvador.
Abstract The objective of this study is to analyzethe intervention strategies presented for the street food segment, based on national and international studies and reports. According to the literature, it is observed a broad character of these intervention strategies, including actions directed to sellers, consumers, public human resources and to the development of appropriate technologies. In relation to the vendors, the strategies highlight the necessity of the activity regulation, the establishment of sanitary regulations, guidelines or codes to the activity as well as training of food handlers and vendors. To consumers, the actions comprise mainly the implementation of educational programs. At public administration sphere the strategies turn to human resources capacitating. Considering the appropriate technologies two possibilities are identified: the construction of specialized street food centers and the improvement of existing stalls. O ne can conclude that given the increase in sales of street food and the potential sanitary risks inherent to this sector, studies point to strategies to organize this segment, which can subsidize the development of social policies for this area in Brazil.

Key words Street food, Legal intervention, Food security, Health surveillance
Resumo Este trabalho tem por objetivo abordar estratégias de intervenção apresentadas para o segmento decomida derua, com baseem estudos erelatos nacionais e internacionais. Segundo a literatura, verifica-se um caráter abrangente das estratégias de intervenção, contemplando ações voltadas para vendedores, consumidores, recursos humanos da administração pública e para o desenvolvimento de tecnologias apropriadas. Em relação aos vendedores, as estratégias evidenciam a necessidade de regulação da atividade, do estabelecimento de normas, diretrizes ou códigos sanitários para a atividade e do treinamento para manipuladores e vendedores. Para os consumidores, as ações compreendem principalmente o desenvolvimento de programas educativos. $\mathrm{N}$ a esfera da administração pública, as estratégias voltamse para a capacitação de recursos humanos. Q uanto às tecnologias apropriadas, são identificadas duas possibilidades: a construção de centros especializados para venda da comida de rua e a melhoria dos pontos de venda já existentes. Conclui-se que, diante da expansão do comércio de comida de rua e do potencial derisco sanitário inerente, a literatura aponta estratégias para organização do setor, quepodem subsidiar o desenvolvimento de políticas sociais para 0 segmento no Brasil.

Palavras-chave Alimentos de rua, Intervenção le gal, Segurança alimentar e nutricional, Vigilância sanitária 
Introdução

0 termo "comida de rua" tem sido utilizado para designar alimentos e bebidas vendidos em vias públicas, destinados ao consumo imediato ou posterior, porém que não necessitam de etapas adicionais de processamento ${ }^{1}$.

Ao longo dos tempos, a venda de alimentos de rua tem se configurado como uma atividade de importância social, econômica, sanitária enutricional. Principalmentenos países em desenvolvimento, este comércio constitui relevante fonte de renda, sendo favorecido pelos elevados índices de desemprego, escassez de postos de trabal hos formais, baixo poder aquisitivo da população, acesso limitado à educação e ao mercado de trabal ho formal, além das migrações da zona rural para a urbana, em virtude da degradação das condições de vida no campo ${ }^{2,3}$.

No Brasil, de acordo com estatísticas do Instituto Brasileiro de Geografia eEstatística4, em 2003, o mercado de trabal ho vem apresentando um aumento da informalidade em áreas urbanas, com a inserção de quase 14 milhões de pessoas no setor. $N$ ão foram localizados dados específicos sobre a dimensão do segmento de comida de rua, mas segundo levantamentos realizados em países da América Latina, a renda obtida na atividade com a venda de alimentos de rua chega a ser de três a dez vezes superior ao salário mínimo vigente nos diferentes países ${ }^{2,5}$, o que contribui para a redução da pobreza. Contudo, é observada ainda uma associação entre informalidade, menor renda e pior situação de saúde ${ }^{3,6}$.

Em relação ao aspecto nutricional, a comida de rua também constitui um reflexo da condição econômica e social do país, na medida em que delineia uma alternativa alimentar e nutricional de fácil aquisição, tanto pela acessibilidadefísica como social, devido ao seu menor custo.

Como resposta ao modo de vida urbano contemporâneo, caracterizado pela escassez de tempo para preparo econsumo dealimentos, pelo deslocamento das refeições de casa para alimentação fora deste ambiente, pelo uso de alimentos prontos e diversificados para o consumo e, ainda, pelaflexibilização nos horários das refeições ${ }^{7}$, a comida de rua compreende a opção mais viável para grande parte da população. Para Tinker ${ }^{8,9}$, o segmento de comida de rua substitui as cadeias de fast-food em muitos países do terceiro mundo, com atendimento destacado para grupos de menor poder aquisitivo.

Segundo estimativas, cerca de 2,5 bilhões de pessoas em todo o mundo consomem diariamente comida de rua ${ }^{10}$. No Brasil, segundo a Pesquisa de Orçamento Familiar ${ }^{11}$, 24,05\% das despesas com alimentos são destinadas a alimentos fora de casa; entretanto, não há registros sobre quanto representa a despesa específica com a comida de rua.

Nessa perspectiva, ainda que a utilização da comida de rua seja histórica no país, remontando à época do escravagismo ${ }^{12}$, verifica-se na atualidade, além do comércio de produtos típicos, a incorporação de novas práticas alimentares, resultantes do que vem sendo denominado de "mundialização da cultura", facilitada pelo caráter permeável da cultura brasileira e evidenciada pela aquisição de alimentos característicos de outras culturas, como a americana e a européi $a^{7}$.

Em muitos casos e para diferentes faixas etárias, estes alimentos têm substituído refeições regulares dos consumidores, cumprindo as funções deatendimento aosrequerimentos de energia enutrientes, para que os indivíduos possam realizar as suas atividades diárias ${ }^{13-15}$.

No entanto, sob a ótica da segurança alimentar e nutricional, entendida como a realização do direito de todos ao acesso regular e permanente a alimentos de qualidade, em quantidade suficiente, sem comprometer 0 acesso a outras necessidades essenciais, tendo como base práticas alimentares promotoras de saúde, que respeitem a diversidade cultural e que sejam social, econômica e ambientalmente sustentáveis ${ }^{16}$, o comércio de alimentos de rua apresenta faces contraditórias: ao mesmo tempo em que pode permitir às parcel as da população acesso ao trabalho, renda e melhor qualidade de vida, também pode torná-las vulneráveis e vítimas do próprio desconhecimento quanto aos cuidados higiênicos com os alimentos, passíveis detransmitirem diversos patógenos ${ }^{17-19}$.

De acordo com estudos desenvolvidos em vários países, a contaminação microbiana destes produtos éfato incontestável, sendo identificada a veiculação de microrganismos como Escherichia coli, Staphylococcus aureus, Clostridium perfringens, Salmonella spp, Vibrio cholerae, entre outros ${ }^{2,17-20}$.

Neste contexto, diferentes aspectos são considerados, incluindo a higiene dos pontos de venda, a procedência da água para limpeza dos utensílios e para preparação dos alimentos, os cuidados adotados nos núcleos de preparo dos al imentos, a forma de conservação, proteção contra vetores e 0 modo como são descartados os resíduos sólidos e líquidos resultantes da atividade ${ }^{13,21}$.

No Brasil, esta prática encontra-se estabelecida nas mais distintas regiões, com o comércio de produtos industrializados e manufaturados, destacando-se preparações da culinária regional e local, que ocupam as ruas das grandes cidades brasileiras; como 
exemplos, o tacacá paraense, a tapioca potiguar, 0 queijo de coalho pernambucano, o acarajébaiano, 0 espetinho carioca e o cachorro quente paulista.

Embora este comércio esteja sujeito à regulação em países desenvolvidos, registra-se uma lacuna normativa em diversos países tropicais. De acordo com levantamentos apresentados pela $\mathrm{Or}$ ganização das Nações Unidas para Agricultura e Alimentação (FAO), na América Latina, a normalização ainda está em evolução; al guns países, como a Bolívia, Colômbia, Peru e Equador, contam com resoluções específicas, considerando os principais aspectos do comércio ambulante de alimentos ${ }^{2}$.

No Brasil, até 2004, não havia legislação federal para a atividade. Ao mesmo tempo, com a implantação do Sistema Ú nico de Saúde e a descentralização das suas ações, o controle sanitário deste segmento passou a ser responsabilidade dos municípios. Desta forma, enquanto al guns municípios avançaram na elaboração de normas próprias, como Curitiba, Natal e São Paulo, muitos sequer alcançaram a organização dos seus serviços de Vigilância Sanitária ${ }^{5,22}$.

N esse contexto, nas mais diversas esferas da administração pública brasileira, observa-se a ausência de ações ou movimentos tímidos em relação a iniciativas de apoio ao comércio de comida de rua, sendo o Programa Alimentos Seguros (PAS), um marco ${ }^{23}$. Como exemplo, na Bahia, em 2002, 0 PAS conduziu o Programa Acarajé 10, que incluía desde a realização de cursos de boas práticas na produção de alimentos até a certificação profissional, e contou com a participação de 109 baianas $^{24}$.

No ano de 2005, a partir da ocorrência de um surto de doença de Chagas associado à venda de caldo de cana, em Santa Catarina, no qual 31 pessoas foram acometidas e cinco foram a óbito ${ }^{25}$, a Agência Nacional de Vigilância Sanitária (ANVISA)/M inistério da Saúde editou, após Consulta Pública, a primeira norma relativa à comercialização de alimentos de rua, a RDC 218/200526. Essa legislação, embora signifique um avanço no sentido do reconhecimento e da preocupação do poder público para a questão, exibelimitações, posto que corresponde a um modelo reativo de ação em saúde. À época, a combinação dos fatores pressão sobre órgãos oficiais da área de saúde e insuficiência de informações sistematizadas sobre o setor, que subsidiassem a reflexão e proposição de um documento mais coerente à realidade brasileira, resultou na obtenção de um conteúdo normativo restrito, tendo em vista a natureza complexa que o segmento de comida de rua descreve em todo o país.

De forma inversa à situação brasileira, países africanos, asiáticos ou ainda da América Latina re- gistram estudos diagnósticos e o estabelecimento de programas de intervenção para o segmento de comida de rua. Estas estratégias, que visam à segurança alimentar pela produção e comercialização dealimentosseguros, com qualidadenutritivaepelo fomento à economia, geralmente são implementadas pela colaboração entre órgãos locais e organismos internacionais de assistência 2,15,27-29.

Nesse contexto, questões sobre a estrutura, organização e medidas de caráter público emergem, tais como: que órgão éresponsável pelo setor de comida derua nos municípios ecomo (ese) são aprovisionadas licenças; que legislação tem sido elaborada e revisada para assegurar a sua regulação; que programas de inspeção e amostragem têm sido implementados; que programas de treinamento para vendedores einspetores têm sido estabelecidos ecom qual efetividadee, por último, que mecanismos de interação são conduzidos entre os diferentes departamentos municipais que atuam no setor ${ }^{29}$.

Paralelamente, considerando o grande número de consumidores urbanos que contam com a comida de rua como parte da sua refeição diária e tendo em vista a natureza perecível de muitos alimentos, além do fato destes se encontrarem em um estado pronto para consumo, sem que o usuário utilize qualquer tratamento que sirva para reduzir os níveis de contaminação, torna-se imperativo o controle pelos órgãos oficiais para proteger a saúde coletiva ${ }^{29}$.

Tomando por base a comida de rua como um segmento da cadeia de suprimento de alimentos para populações urbanas e as projeções de crescimento populacional para as cidades de médio e grande porte no Brasil ${ }^{30}$, as questões ambientais e de saúde pública relativas a este comércio tendem a piorar, na ausência de planejamentos efetivos ${ }^{29}$.

M ediante a consolidação deste segmento e 0 interesse de organismos como a Organização M undial de Saúde (OMS), a Organização Pan-Americana deSaúde(OPAS) ea FAO em estabelecer programas e recomen dações para o alcance de melhores níveis de saúde para populações, pela prática da alimentação saudável, prioritário se faz assegurar a formulação, implementação e avaliação de políticas públicas que visem à aquisição e consumo de alimentos seguros.

Ressalta-se que o enfoque prioritário conferido para a questão da segurança dos alimentos pelo governo e autoridades locais constitui fator crítico para o sucesso de qualquer programa de intervenção, na medida em que há a exigência de recursos significativos e de suportetécnico epolítico desdeo nível central de governo ${ }^{28}$. N este sentido, o Brasil 
experimenta um cenário político extremamente favorável para a produção de conhecimentos e de tecnologias de intervenção neste campo, uma vez que a segurança alimentar ocupa na atualidade 0 topo da agenda nacional.

Desta forma, este trabalho busca abordar propostas e estratégias deintervenção para o fenômeno social da comida de rua, relatadas a partir de estudos e experiências conduzidas em países do mundo em desenvolvimento, contemplando uma descrição quanto aos diferentes elementos envolvidos.

\section{Procedimentos metodológicos}

Trata-se deum estudo derevisão bibliográfica, com tema na área de segurança alimentar (food safety), mais especificamente voltado para a questão da comida de rua, na dimensão das estratégias de intervenção.

Foram selecionadas publicações divulgadas a partir de 1994, identificadas nas bases de dados SCIELO, LILACS, M ED LINE eEPIN et, complementadas com referências da FAO, OMS e de outras organizações de assistência internacional, bem como de órgãos e institutos no Brasil.

As palavras-chave, com os respectivos termos em inglês ou espanhol, utilizadas para a revisão compreenderam: comida ou alimentos derua (stre et food/alimentos callejeros), segurança alimentar (food safety), alimentos (food), doenças transmitidas por alimentos (foodborne disease), higiene(hygiene), qualidade (quality/calidad), estratégias (strategy/estrategias) emelhoria (improvement/mejora).

\section{Propostas de intervenção}

Propostas de intervenção para o segmento de comida de rua, relatadas com base na experiência de diferentes países em cooperação com organismos internacionais de assistência, têm orientado o planejamento de ações para o setor a partir de dados diagnósticos ${ }^{2,28,33}$.

Assim, concebendo o segmento do comércio informal de alimentos de rua como uma temática em que a escassez de dados expressa o pouco conhecimento da realidade, pretende-se, com esta avaliação de situação, obter informações para facilitar decisões políticas e o planejamento estraté gico para o setor ${ }^{29}$. Paralelamente, espera-se que as informações possam contribuir para avaliar o impacto das medidas a serem tomadas e os seus desdobramentos, nas dimensões socioeconômica, nutricional e de segurança, atentando para a população beneficiada por este comércio.

Estratégias de intervenção para melhoria da qualidade da comida derua, além de requererem 0 conhecimento prévio da atividade, abarcam os seguintes pressupostos ${ }^{15,29,32:}$

- primeiro, que a intervenção não deve ocorrer concomitanteà etapa de diagnóstico do estudo, de modo a evitar erros de tendência;

- segundo, que o fenômeno da comida de rua deve ser reconhecido pelas autoridades governamentais, representando um espaço legítimo nas cidades, sob diversos aspectos, pois somente a partir destereconhecimento tornam-se possíveis mudanças de atitudee o estabelecimento de políticase estratégias deintervenção, bem como a redução de medidas repressivas sobre os vendedores;

- terceiro, quea temática da comida de rua deve estar efetivamente inserida em uma política em nível central, para que haja apoio contínuo e que as ações propostas tenham sustentabilidade e efetividade;

- quarto, que há a necessidade de ações intersetoriais, envolvendo diferentes esferas da administração pública relacionadas ao segmento, de modo a abranger todos os elementos que o integram, como exemplo, setores referentes às áreas social, econômica, de saúde e de ordenamento e uso do espaço público urbano.

Segundo Clarke ${ }^{29}$, tomando por base uma visão holística para organização e melhoria do segmento da comida rua, propostas de intervenção devem observar um conjunto de aspectos, que inclui: a necessidade dos habitantes urbanos de disporem de alimentos acessíveis e de baixo custo; 0 estilo de vida de setores vulneráveis da população que fazem uso da comida de rua; os recursos requeridos para a efetiva regulação do setor; os custos estimados para a adequada provisão de serviços; o uso competente dos recursos e o impacto total para a comunidade, em virtude da inadequada regulação do setor.

\section{Objetos de intervenção}

Considerando a relação de compra evenda e o uso do espaço público, para a manutenção do segmento de comida de rua, identificam-se, na literatura, estratégias de interven ção com programas direcionados tanto para vendedores e consumidores quanto para servidores públicos que atuam nesta área $2,15,28,29,32,33$. 
M etodologias de intervenção para vendedores

Regulação

A regulação da venda de comida nas ruas se configura como um dos maiores avanços entre as estratégias propostas para o setor, tanto no que se refere ao uso ordenado do espaço público quanto no que trata do controle sanitário, tendo em vista a complexidade dos aspectos relacionados à conformação e à continuidade do segmento.

Embora a decisão de regulação (se expede ou não licenças, para quetipo de produto e como isto se dá) constitua uma das questões mais difíceis no delineamento do processo de intervenção, representa também uma base legal para o reordenamento do comércio de comida de rua e um apoio ao processo de descentralização de ações em saúde pública, gerando ou consolidando regulamentos municipais com base em normas nacionai $5^{2,31}$.

Deacordo com análises desituações e considerando a relação custo/efetividade, experiências apontam para o princípio de permitir a atividade para o segmento, mas controlar as condições sob as quais el efunciona. Assim, sob o aspecto regulatório, as licenças podem trazer especificações, como: alimento(s) para qual(is) o documento é válido, local em que será preparado e comercializado e prazo de vigência ${ }^{15,31}$.

A regulação significa também colocar a comida de rua sob parâmetros específicos, podendo estabelecer restrições locais onde os alimentos poderiam ser preparados e vendidos ou uma delimitação de centros de venda ou centrais de higienização. N este contexto, espaços próprios devem ser planejados e oferecidos, atendendo requisitos de funcionamento como o suprimento deágua eenergia, infra-estrutura e instal ações sanitárias e serviço de coleta de lixo ${ }^{31}$.

$\mathrm{N}$ o intuito deassegurar maior exequibilidadee efetividade para as decisões, reforça-se a necessidade de participação essencial de representantes dos vendedores e de consumidores e de transparência no processo ${ }^{31}$.

Elaboração de normas, diretrizes e códigos sanitários

Associada ao processo de regulação, a intervenção requer o estabelecimento de normas, diretrizes ou códigos sanitários para orientar as práticas inerentes à produção e comercialização de alimentos de rua, bem como respaldar o processo de controlee inspeção ${ }^{15}$.
Levantamentos sobre regulação em países com experiências de intervenção evidenciam leis distintas, em estruturas variadas e com diferentes impactos $^{33}$. Contudo, grande ênfase tem sido colocada no cuidado para não haver uma regulação excessiva e repressão desta alternativa alimentar, tendo em vista os riscos destas medidas aumentarem os índices de desnutrição e de fome $e^{15,34}$. Ao mesmo tempo, 0 aumento do rigor no cumprimento da lei ea apreensão de produtos e estruturas de venda apresentam resultados insatisfatórios, visto que estecomércio logo se restabelece, com o apoio da população ${ }^{33}$.

Observando a diversidade de produtos e preparações neste segmento, bem como a dificuldade de regulação, comitês assessores da FAO, OMS e OPAS têm sistematicamente sugerido o uso de um código de práticas de higiene e segurança para comida de rua, em substituição às leis e normas tradicionais. Este código, geralmente recomendado por autoridades nacionais em segurança dealimentos, pode sofrer modificações por autoridades locais, quando necessário, para 0 atendimento de necessidades específicas ${ }^{31}$.

Documentos fornecendo orientações quanto à conformação e conteúdo para este código, incluindo a adoção de princípios do sistema de Análise dePerigosePontos Críticos deControle(APPCC), são apresentados nas publicações Essential safety requirements for street-vended foods ${ }^{1}$ e Revised code of hygienic practice for the preparation and sale of street food (Regional code - Latin America and the (aribean) $)^{35}$.

Treinamento para vendedores e manipuladores

A capacitação de vendedores e manipuladores de alimentos de rua tem se constituído em um suporte imprescindível para alcançar mudanças de atitude emelhoria das condições de preparo evenda destes alimentos, pois somente por meio deste treinamento e subsequente monitoramento da situação é que vendedores poderiam ser integrados e considerados como uma parte responsável pelo sistema de suprimento alimentar da cidade².

No entanto, em virtude da natureza transitória de al guns vendedores, das longas horas de atividade e da limitação de recursos financeiros para promover mudanças e assegurar a sanidade dos alimentos, este trabalho, muitas vezes, representa um desafio ${ }^{33}$.

Vendedores e manipuladores necessitam ser treinados em cursos e durante visitas às suas instalações. As estratégias de treinamento têm incluído lições ao ar livre, exposições, cartazes, represen- 
tações na rua, projeções de vídeos, debates, cursos para formação de multiplicadores, sessões orientadas individualmente e programas educacionais públicos com uso da mídia de massa. Adicionalmente, a utilização de folhetos ilustrados, evidenciando práticas aceitáveis, fixados próximos a vendedores e consumidores, contribui para que ambos possam estar atentos, 233,36.

Neste contexto, tanto os cursos quanto os materiais desenvolvidos têm considerado as idiossincrasias dos vendedores, suas características socioculturais e seus valores e conceitos em relação à higiene dos alimentos e têm promovido a sua própria revalorização em relação ao papel que repre sentam para a sociedade?

0 desenvolvimento de treinamentos em cooperação com associações comerciais de vendedores, quando elas existem, ou mesmo com a participação de organizações não governamentais e do setor privado facilitam estatentativaeestimulam esta açãa ${ }^{31,33}$.

Estudos conduzidos no Peru e na Colômbia têm demonstrado que vendedores de comida de rua desejam e estão dispostos a aceitar o treinamento em higiene, com o propósito de produzirem alimentos melhores e mais seguros. Nestes países, materiais de treinamento têm sido elaborados eutilizados; contudo, poucos países da América Latina desenvolveram ações neste sentido, havendo muito a ser realizado ${ }^{33}$.

\section{M etodologias deintervenção para consumidores}

Com o seu poder de compra, o consumidor fornece, talvez, a mais forte motivação para modificar práticas de mani pulação de alimentos, pois, de fato, é ele que faz a escolha do que consome, do lugar onde vai adquirir o produto e que sofre as consequências caso o alimento seja inseguro.

Assim, na medida em quea qualidade de serviços eprodutos fornecidos refleteo nível de exigência dos seus consumidores, torna-se essencial orientar consumidores de comida de rua para exerce rem um papel mais atuante, com vistas ao alcance e manutenção da prestação de serviços com me Ihor qualidade.

N este cenário, programas educativos para sensibilizar consumidores constituem um complemento ao treinamento dos vendedores, pois, na medida em que os primeiros tornam-se cientes da sua responsabilidade pela qualidade demandada, convertem-se em um dos principais aliados das autoridades de saúdereguladoras eagente essencial para mudanças de atitudes dos vendedores ${ }^{32}$.
Desta forma, alguns países têm desenvolvido campanhas para sensibilizar os consumidores sobre a importância da sua participação para meIhorar a qualidade dos alimentos derua. Com base em características e percepções dos consumidores quanto à higiene de alimentos, as campanhas divulgam mensagens educativas utilizando veículos de mídia, principalmente televisão, rádio e meios impressos; outros instrumentos de educação compreendem cartazes e folders, que são afixados edistribuídos em diversos pontos das cidades ${ }^{2,31}$.

Empreendendo uma postura mais arrojada, associações regionais de consumidores e organizações não governamentais têm assumido o propósito de desenvolver ações para orientar a população e promover a inocuidade e a qualidade dos alimentos em geral, destacadamente dos que são vendidos nas ruas 2 .

Considerando, ainda, o grande nível de desinformação em educação sanitária da população, o desconhecimento da associação entre a contaminação de alimentos e a ocorrência de doenças veiculadas por alimentos e o amplo uso da comida de rua por indivíduos em diversas faixas etárias, preconiza-se, de modo prioritário, a integração de conteúdos de higiene de alimentos em programas deeducação dosníveis fundamental emédio $0^{2,23,31,33}$.

M etodologia de intervenção para recursos humanos da administração pública

N a complexa questão da comida de rua, estratégias de intervenção devem ser dirigidas para os diferentes níveis hierárquicos da administração públi$\mathrm{ca}$, envolvendo desde coordenadores da área de controle de alimentos até funcionários em níveis operacionais².

Com relação aos programas para coordenadores, a capacitação orienta-se para a gestão de programas integrais de controle sanitário de alimentos, para o papel do gestor de alimentos, assim como enfoca mecanismos de programação e avaliação de ações e a inclusão do controle dos alimentos de rua nesses programas².

Em referência aos inspetores efuncionáriosque lidam com o segmento, deve-se buscar, a priori, uma mudança de atitude, em relação aos vendedores e aos consumidores. N este sentido, espera-se que os funcionários, principal mente os inspetores, passem a agir como aconselhadores na promoção de uma mudança de comportamento dos vendedores e consumidores, em vez de se perceberem meramente como oficiais exercendo funções repres$\operatorname{sivas}^{15,32}$. Assim, o treinamento representa uma es- 
tratégia essencial para aprimorar as atividades dos funcionários na prática com a comida de rua.

0 treinamento deve ser realizado contemplando diferentes aspectos, como: princípios de microbiologia de alimentos e segurança alimentar, perigos microbiológicos e suas soluções, tecnologias para alimentos processados, pontos críticos de controle (PCCs), critérios e procedimentos de monitoramento e ações a serem tomadas quando PCCs estão fora de controle, com destaque para estudos especiais conduzidos sobre alimentos de alto risco e elevado consumo $0^{2,28,33}$.

Este treinamento, desenvolvido em uma sistemática de capacitação de multiplicadores, deve estar apoiado em instrumentos técnicos como códigos de práticas, manuais básicos, formulários de inspeção e de avaliação das mudanças ocorridas e outros tipos de recursos educacionais $s^{2,31-33}$.

Limitações de práticas correntes enfatizando perigos microbiológicos e não aspectos estéticos, reações defensivas diversas, ensino de técnicas de abordagem e comunicação e o estímulo de trainees para a tomada de decisão também devem ser abordados 2,33 .

Em al guns países latinos, ainda, foi verificada a necessidade da capacitação de recursos humanos de laboratórios, enfocando a identificação de microorganismos indicadores para os alimentos e a sua interpretação, a revisão de metodologias para amostragem e o controle de qualidade para estes serviços².

$\mathrm{Na}$ Índia, por outro lado, oficiais de polícia foram sensibilizados sobre a sua responsabilidade em relação à comida de rua de Calcutá, em uma mobilização com outras instituições, criando condições para a adoção de uma política relativa aos alimentos de rua da cidade ${ }^{36}$.

Como forma de dar direção à atividade de segurança alimentar, se recomenda, ainda, que levantamentos de doenças veiculadas por alimentos sejam promovidos e utilizados ${ }^{33}$.

\section{Desenvolvimento detecnologias apropriadas}

Os pontos de venda de alimentos de rua compreendem uma ampla diversidade de formas, dimensões e materiais, que incluem desde simples sacos, caixas e mesas até instalações mais organizadas, contando com estrutura de metal, fornecimento de energia, água e refrigeração. Entre estes extremos, estão diferentes model os que respondem pelos valores étnicos e culturais locais, expectativas dos consumidores edisponibilidade financei ra dos vendedores ${ }^{21}$.
Sob o aspecto de preparo dos alimentos nas ruas, a infra-estrutura adotada pelos vendedores corresponde, em geral, a três situações: alimentos preparados em casa, alimentos preparados na rua e alimentos que são semipreparados em casa ecom preparo final nos pontos ${ }^{21}$.

Quase sempre, contudo, a improvisação tem caracterizado as diversas formas dos pontos de venda e desconsiderado requisitos sanitários mínimos para o preparo e a comercialização de alimentos nas ruas ${ }^{21}$. N este contexto, ressalta-se que o setor de comida de rua envolve muito trabalho informal, baixo nível de tecnologia, investimento mínimo de capital e limitado conhecimento sobre higiene, existindo para muitos vendedores a simples transferência do preparo doméstico dealimentos para o preparo em espaços públicos ${ }^{31}$.

Assim, a incorporação de tecnologias apropriadas para o preparo ea venda dos alimentos constitui uma das principais linhas de ação nos projetos desenvolvidos para melhorar a qualidade sanitária e agregar valor aos produtos, promovendo 0 setor.

Trabalhos visando à busca de tecnologias meIhoradas têm considerado aspectos críticos do comércio de alimentos nas ruas, incluindo: 0 suprimento de água potável; os utensílios e equipamentos utilizados para cocção, estocagem e venda; meios de aquecimento eresfriamento efontes de energia; o desenho das instalações; os invólucros e as embalagens utilizadas para os alimentos; a forma deservir; procedimentos delimpeza ede disposição do lixo e a provisão de instalações sanitárias ${ }^{21,31}$. Paralelamente, apesar da amplitude das demandas apontadas, constitui fato reconhecido que mesmo pequenas melhorias, para qualquer um dos aspectos apresentados, contribuiria para melhorar o serviço de atendimento às necessidades de milhões de pessoas que dependem da comida de rua, beneficiando vendedores e consumidores ${ }^{31}$.

Avanços quanto à adoção de tecnologias meIhoradas são observados em algumas localidades da Índia, pela prática da transferência ou da concentração de ven dedores em centros especialmente desenhados, dotados de fornecimento de água potável eeletricidade, serviços de disposição e coleta de lixo e de instalações sanitárias, havendo, ain$d a$, a descrição de centros que dispõem de facilidades de aquecimento e congelamento para alimentos de al to risco, sob supervisão de autoridade sanitária. Este modelo, contudo, em virtude do custo dos centros e da transferência dos vendedores, não são viáveis para muitos municípios ${ }^{31}$.

$\mathrm{Na}$ América Latina, a adoção de tecnologias apropriadas é concebida sob duas formas: solu- 
ções integrais de todos os problemas, substituindo o ponto de venda, ou a modificação parcial dos pontos já existentes, aos quais se agregam gradativamente as soluções para cada aspecto deficiente, seguindo uma ordem de prioridade e de acordo com as possibilidades econômicas do vendedor. Em ambos os casos, se torna necessário o respaldo de uma instituição de crédito, que financie em condições favoráveis eacessíveis à maioria dos vendedores, tendo as instituições técnicas de cooperação e as organizações não governamentais um papel-chave nesta área21.

A busca de soluções integrais tem conduzido ao desenvolvimento de diversos modelos de pontos de venda. Construídos em metal, utilizando aço inoxidável para a superfície de trabalho e polivinil ou fibra de vidro para os depósitos de água, representam, em geral, estruturas muito caras. No caso das soluções parciais, a dotação de água foi uma das prioridades, sendo desenvolvidos diferentes modelos de depósitos, sendo o mais popular um modelo de 40 litros que se adéqua à recomendação do código de práticas higiênicas do Codex Alimentarius ${ }^{21}$.

A opção de adoção entre a solução integral ou parcial está relacionada com o tamanho do negócio, evidenciando que os esforços para melhoramento da estrutura tendem a ter êxito na medida em que a atividade seja rentável. Os vendedores aceitam investir em melhorias, principalmente quando constatam que resultam em benefícios para as suas vendas. Deste modo, a maior limitação para a adoção de tecnologias apropriadas nos pontos de venda parece ser de ordem econômica, o que pode ser superado quando se dispõedeoportunidades de crédito para o seu financiamento ${ }^{21}$.

\section{Conclusões}

Abordar estratégias de intervenção para o segmento de comida de rua constituiu a proposta deste trabalho. Nesse sentido, parte-se de uma caracterização do objeto, na qual são evidenciados aspectos positivos e negativos, na perspectiva da segurança alimentar e nutricional, e são colocadas sob reflexão questões anteriores à própria intervenção, a saber: a necessidade de estudos diagnósticos e a decisão política do poder público, em nível central, de reconhecer o segmento e de apoiar o desenvolvimento deações intersetoriais sustentáveis, visando à efetividade das estratégias de intervenção e melhoria deste comércio.

Considerando as diferentes dimensões e elementos que compreendem o fenômeno da comida de rua, o relato de organismos internacionais de assistência desvenda um caráter abrangente das estratégias deintervenção dirigidas para o segmento, contemplando vendedores, consumidores, recursos humanos da administração pública e o desenvolvimento de tecnologias apropriadas.

Em relação aos vendedores, as estratégias apontam a necessidade de regulação da atividade, entendida como um avanço no processo de intervenção e um apoio na sistemática de descentral ização das ações de saúde, com o estabelecimento de normas, diretrizes ou códigos sanitários que possam orientar vendedores e inspetores e o treinamento para manipuladores e vendedores. São destacadas a preocupação quanto à possibilidade de superregulação, com desdobramentos desfavoráveis ao próprio segmento eà população atendida, a orientação quanto à adoção de princípios do sistema APPCC na elaboração dos códigos sanitários, e a essencialidade do treinamento, observando características locais, o potencial de uso dos recursos didáticos e a articulação com associações de vendedores e organizações não governamentais.

Para os consumidores, as ações compreendem principalmente programas educativos em higiene de alimentos, utilizando diferentes veículos de comunicação ea rede de educação formal, buscando uma sensibilização para o desenvolvimento deuma postura mais exigente quanto à qualidade do serviço prestado.

Na esfera da administração pública, as estratégias voltam-se para a capacitação de recursos humanos nos diferentes níveis hierárquicos e setores quelidam com o segmento. N esseâmbito, são destacados o papel dos gestores, enquanto controladores da qualidade dos alimentos, e a capacitação teórica e de conduta de inspetores, nas suas rotinas com a comida de rua, buscando realizar práticas de aconsel hamento com vistas à mudança de atitudes de vendedores e de consumidores.

Em referência ao desenvolvimento de tecnologias apropriadas, relatos de experiências em diferentes continentes evidenciam fundamentalmente duas possibilidades: a construção de centros especializados para venda da comida de rua e a melhoria dos pontos de venda já existentes, compreendendo benfeitorias parciais ou mesmo a substituição integral da estrutura de venda. Para qualquer uma das soluções apresentadas, é ressaltada a bai$x a$ acessibilidade econômica dos vendedores às novas tecnologias, sendo importante a participação de instituições técnicas de cooperação e organizações não governamentais neste processo.

$\mathrm{Na}$ medida em que se observa a consolidação da comida de rua, o potencial de risco sanitário 
associado a este comércio, bem como a insuficiência de trabalhos nestaárea, no Brasil, espera-se que novos estudos possam ser apresentados, de modo a contribuir para a discussão e orientação de estratégias a serem futuramente adotadas no país.

\section{Colaboradores}

RCV Cardoso coordenou o levantamento e a sistematização das referências e a organização da publicação. SM C Santos e EO Silva contribuíram na sistematização das referências e na revisão e análise do texto.

\section{Referências}

1. World Health Organization. Division of Food and Nutrition. Essential safety requirements for street-vended foods. (Revised edition). 1996. [cited 2002 Jun 18]; [about 36 p.]. Available from: http://www.who.int/ fsf/96-7.pdf

2. Costarrica ML, Morón C. Estrategias para el mejoramiento de la calidad de los alimentos callejeros en América Latina y en el Caribe. In: Albert JL, editor. Food, Nutrition and Agriculture [serial on the Internet] 1996 [cited 2002 Jun 18]; [about 21p.]. Available from: http://www.fao.org/WAICENT/FAOINFO/ ECONOMICESN/fnal7-e/sum-e.htm

3. Ulissea G. Informalidade no mercado de trabalho brasileiro: uma resenha da literatura. Rev Econ Pol. 2006; 26(4):596-618.

4. Instituto Brasileiro de Geografia e Estatística. Comunicação Social. Economia Informal U rbana - 2003. [acessado 2007 ago 07]; [cerca de 4 p.]. Disponível em: http://www.ibge.gov.br/home/presidencia/noticias/noticia_impressao.php?id_noticia $=366$

5. Germano $\bar{M} I P$, Germano $P M \bar{L}$, Castro AP, Andrighetto C, Babadopulus P, Koshio S, Pedro SCM, Colombari V. Comida de rua: prós e contras. Hig Alim. 2000; 14(77):27-33.

6. Giatti L, Barreto SM. Situação do individuo no mercado de trabalho e iniqüidade em saúde no Brasil. Rev. Saude Publica 2006; 40(1):99-106.

7. Garcia RWD. Reflexos da globalização na cultura alimentar: considerações sobre as mudanças na alimentação urbana. Rev. Nutr. 2003; 16(4):483-492.

8. Tinker I. Legalizing street foods in the third world; the right to eat on the street. Whole Earth Rev [serial on the Internet] 1989 [cited 2005 Apr 22]; [about 5 p.]. Available from: http://findarticles.com/p/articles/ mi_m1510/is_n62/ai_7422473

9. Tinker I. Street foods into 21st century. Agriculture and Human Values 1999; 16(3):327-333.

10. Food and Agriculture Organization of the United Nations. News \& Highlights. Street foods made safer. 2001. [cited $2003 \mathrm{Jul} 24$ ]. [about 2 p.]. Available from: http://www.fao.org/news/2001/010803-e.htm

11. Instituto Brasileiro de Geografia e Estatística. Pesquisa de Orçamentos Familiares 2002-2003 - Comentário dos Resultados. [acessado 2007 ago 07]; [cerca de 52 p.]. Disponível em: http://www.ibge.gov.br/home/ estatistica/populacao/condicaodevida/pof/2002/ comentario.pdf

12. Zarvos M, Ditadi CAS. Multissabores: a formação da gastronomia brasileira. Rio de Janeiro: SENAC Nacional; 2000. 
13. Garcia-Cruz CH, Hoffmann EL, Bueno SM. Monitoramento microbiológico de lanches vendidos por ambulantes na parte central da cidade de São José do Rio Preto, SP. Hig Alim 2000; 14(75):48-51.

14. Riet $H, H$ artog $A P$, M wangi $A M, M$ wadime RKN Foeken DWJ, Staveren WA. The role of street foods in dietary pattern of two low-income groups in Nairobi. Eur J Clin Nutr 2001; 55:562- 570.

15. Latham MC. Street foods. In: FAO. Food and Nutrition Series. N. 29: Human Nutrition in the developing world. FAO, Rome, 1997. [cited 2002 Jul 14]; [about 7 p.]. Available from: http://www.fao.org/docrep/ w0073e/w0073e07.htm

16. Conselho Nacional de Segurança Alimentar e Nutricional. Princípios e diretrizes de uma política de segurança alimentar e nutricional. 2004 [acessado 2007 jul 15]; [cerca de 80 p.]. Disponível em: http:// www.fomezero.gov.br/documentos

17. Estrada-Garcia T, Cerna JF, Thompson MR, LopezSaucedo C. Faecal contamination and enterotoxigenic Escherichia coli in street-vended chili sauces in M exico and its public health relevance. Epidemiol Infect 2002; 129:223-226.

18. Garin B, Aidara A, Spiegel A, Arrive P, Bastaraud A. Cartel J-L, Aissa RB, Duval P, Gay M, Gherardi C, Gouali M, Karou TG, Kruy SL, Soares JL, M ouffok F, Ravaonindrina N, Rasolofonirina N, Pham MT, Wouafo M, Catteau M, M athiot C, M auclere P, Rocourt J. Multicenter study of street foods in 13 towns on four continents by the food and environmental network of Pasteur and associated institutes. J Food Prot 2002; 65(1):146-152.

19. Mensah P, Yeboah-Manu D, Owusu-Darko K, Ablordey A. Street foods in Accra, Ghana: how safe are they? Bull World Health Organ 2002; 80(7):546-554.

20. Muleta D, Ashenafi M. Salmonella, Shigella and growth potential of other food-borne pathogens in Ethiopian street vended foods. East Afr M ed J 2001; 78(11):576580.

21. Huaman JP. Las tecnologías apropiadas para la venta callejera de alimentos. In: Albert JL, editor. [serial on the Internet] 1996 [cited 2002 Jun 18]; [about 11 p.]. Available from: http://www.fao.org/docrep/W 3699T/ w3699t09.htm

22. Germano PML, Germano MIS. Higiene e vigilância sanitária de alimentos. São Paulo: Livraria Varela; 2001.

23. Serviço Nacional de Aprendizagem Industrial. 0 Programa: Programa Alimentos Seguros - PAS. 2003. [acessado 2003 set 17]; [cerca de 2 p.]. Disponível em: http://www.alimentos.senai.br/subpaginas/projeto.htm

24. Carvalho Filho CD, Guimarães AG, Sobral NAT. Avaliação do Programa Acarajé 10, em Salvador, Bahia. Hig Alim 2003; 19(133):15-19.

25. Brasil. M inistério da Saúde. Secretaria de Vigilância em Saúde. N ota Técnica: Doença de Chagas relacionada à ingestão de Caldo de cana em Santa Catarina. [acessado 2006 mar 22]; [cerca de 2 p.] Disponível em: http://www.anvisa.gov.br/divulga/noticias/2005/ 240305_nota.pdf
26. Brasil. Ministério da Saúde. Agência Nacional de Vigilância Sanitária. Resolução de Diretoria Colegiada - RDC 218, de 29 de julho de 2005. DOU de 1/08/ 2005. Dispõe sobre o Regulamento Técnico de Procedimentos Higiênico-Sanitários para M anipulação de Alimentos e Bebidas Preparados com Vegetais. 2005. [acessado 2005 ago 17]; [cerca de 6 p.]. Disponível em: http://e-legis.bvs.br/leisref/public/ showAct.php?id=18094\& word

27. Natural Resources Institute. Street foods in Africa: Developing food safety strategies and procedures through reduction of food hazards in street-vended foods to improve food security for consumers, street food vendors and input suppliers. 2002. [cited 2003 Set 04]; [about 3 p.]. Available from: http:www.nri.org/streetfoods/ project2.htm

28. Food And Agriculture Organization of The United Nations/ World Health Organization. The experience of improving the safety of street food via international technical assistance: Country paper proposed by China. Marrakech, 2002. [cited 2003 Ago 02]; [about 4 p.]. Available from: http://www.fao.org/docrep/meeting/ 004/ab439e.htm

29. Clarke R. Street foods in Asia: food safety and nutritional aspects. 2000. [cited 2003 Ago 02]; [about 5 p.]. Available from: http://www.fao.org/ag/ags/agsm/sada/ asia/DOCS/DOC/WP B5.doc

30. Instituto Brasileiro de Geografia e Estatística/ Fundo de População das Nações Unidas. Situação da população brasileira - 2002. Rio de Janeiro: IBGE; 2002.

31. Food and Agriculture Organization of the United Nations. Street foods (FAO food and nutrition paper). Roma, 1997. [cited 2003 Set 02]; [about 22 p.]. Available from: http://www.fao.org/docrep/w4128t/ w4128t00.htm

32. Arámbulo III P, Almeida CR, Cuéllar SJ, Belotto $A B$. Street food vending in Latin America. Bull. PAHO 1994; 28(4):344-354.

33. Simopoulos AP, Bhat RV. Street foods. World Rev Nutr Diet 2000; 86:123-137.

34. Moy G, Hazzard A, Käferstein F. Improving the safety of street-vended food. WId HIth Statist Quart 1997; 50:124-131.

35. Instituto Panamericano de Protección de Alimentos. Revised code of hygienic practice for the preparation and the sale of street foods (Regional Code - Latin America and the Caribbean) - CAC/RCP 43-1997, Rev. 1-2001. [cited 2003 Ago 26]; [about 10 p.]. Available from: http://www.panalimentos.org/cclac/files/Codigo $\% 20$ Regional.pdf

36. Food And Agriculture Organization of The United $N$ ations. M ejora de los alimentos callejeros en Calcuta (TCP/IND/0155): Ganadores del Premio Edouard Souma, 1994/1995, s.d. [cited 2002 Jun 18]; [about 2p.]. Available from: http://www.fao.org/tc/tcd/tcdt/ Es/ind0155.htm
Artigo apresentado em 24/08/2006

Aprovado em 25/06/2007

Versão final apresentada em 14/08/2007 